\title{
Using high effective risk of adult-senior duo in multigenerational homes to prioritize COVID-19 vaccination
}

\author{
Brijesh Saraswat $^{1}$, Santosh Ansumali ${ }^{1,2, *}$ and Meher K. Prakash ${ }^{1,3}$ \\ ${ }^{1}$ Jawaharlal Nehru Centre for Advanced Scientific Research, Jakkur, Bengaluru 560 064, India \\ ${ }^{2}$ Sankhya Sutra Labs, Manyata Embassy Business Park, Bengaluru 560 045, India \\ ${ }^{3}$ VNIR Biotechnologies Pvt Ltd, Bangalore Bioinnovation Centre, Helix Biotech Park, Electronic City Phase I, Bengaluru 560100 , India
}

Universal vaccination on an urgent basis is a way of controlling COVID-19 infections and deaths. Vaccine shortage and practical deployment rates on the field necessitate prioritization. The global strategy has been to prioritize those with high personal risk due to their age or comorbidities, and those who constitute the essential workforce of the society. Rather than a systematic age-based rolldown, assigning the next priority requires a local strategy based on vaccine availability, effectiveness of the specific vaccines, population size as well as its age demographics and the scenario of how the pandemic is likely to develop. The adult (2060 yrs) - senior (over 60 yrs) duo from a multigenerational home presents a high-risk demographic. The estimated 'effective age' of an adult living with a grandparent who is not vaccinated may be up to 40 years higher. The proposed model suggests that strategically vaccinating the adults from multigenerational homes in India may be effective in saving the lives of around 70,000 to 200,000 seniors, under the different epidemiological scenarios possible with or without strict lockdowns.

Keywords: COVID-19, high risk groups, intergenerational homes, population demographics, vaccination strategy.

CoviD-19 pandemic management required the use of nonpharmaceutical interventions (NPIs), including severe full or graded lockdowns ${ }^{1}$. The nearly 3 million deaths caused by COVID-19 across the world are much less than the counterfactual scenario with no interventions. Although herd immunity through infection was seen as an option, the unprecedented scale of deaths argued against $\mathrm{it}^{2,3}$. Thus NPIs continue to play a critical role of flattening the infection curve ${ }^{4}$.

The only way of ending the pandemic, and to end the cycle of graded lockdowns is immediate and universal vaccination. Commensurate with the scale of the pandemic, several vaccines have been developed and became available at an accelerated rate ${ }^{5-7}$. They have demonstrated

\footnotetext{
*For correspondence. (e-mail: ansumali@jncasr.ac.in)
}

effectiveness ranging from $60 \%$ to $95 \%$ (ref. 6). Although these vaccines are available and effective in principle, there are several challenges in their deployment. The vaccines based on mRNA technology require a cold chain of $-80^{\circ} \mathrm{C}$, while others with less stringent cold-chain requirements have lower effectiveness. More importantly, all of these vaccines are with a limited production and supply capacity ${ }^{8,9}$ compared to the immediate global need.

Considering these challenges, the taskforces across the world have recommended prioritized vaccination of the vulnerable $^{10}$. The principal factor guiding the recommendations is the mortality risk posed by age (over $60 \mathrm{yrs}$ ) and comorbidities. The priority group also included healthcare workers and other essential workforce of the society, who although may not be at the risk of death, cannot be quarantined due to infections without crippling the functional society. The choice of the vulnerable and the essential for the first priority was almost universally adopted by all the Governments.

However, the strategy for the next phase of prioritization is not obvious. At the rate of current vaccination, it is feared that most countries may need at least another year to vaccinate their entire population, in the ideal scenario without any manufacturing or distribution delays. A systematic roll-down across the different age groups is not a practical strategy for many countries, especially if they have a large young population, such as in India. The 'pyramid' structure of the age demographics in the population, where the population increases in the younger age bracket, presents more candidates to be vaccinated in each roll-down phase. Paradoxically, the younger population which is larger in number also has a lower mortality risk ${ }^{11,12}$, which requires a re-examination of the vaccination strategy beyond those covered in the first priority.

Since the availability of vaccines in USA has not been a challenge, and most European countries are still vaccinating those in the first priority category, it appears that the grey area that is faced by India today on 'who is next?', did not receive sufficient attention. The European countries may also be confronted with this question as soon as the first priority group is vaccinated. 
Vaccinating family members of the vulnerable is a well-known strategy when the vaccine is considered not so safe, or if those vulnerable have lower immunity levels, due to a pre-existing HIV infection or other factors which prevent them from developing antibodies ${ }^{13}$. However, since the safety of all the COVID-19 vaccines has been demonstrated, the discussion of vaccinating the family members of the vulnerable did not gain prime importance.

In this study, we systematically evaluate and demonstrate the effectiveness of this strategy of vaccinating the young family members from multigenerational homes on a priority basis in the Indian context, by combining it with the local realities of vaccination availability, effectiveness as well as the developing epidemiological scenario.

\section{Results and discussion}

\section{Indian COVID-19 and vaccination scenario}

The vaccination strategy should consider the three local realities of the society for which the analysis is done: Vaccine availability, COVID-19 incidence and age demographics.

Vaccine availability: COVISHIELD (Serum Institute of India-AstraZeneca Plc) ${ }^{7}$ and COVAXIN (Bharat Biotech Intl Ltd) ${ }^{14}$ are the first two vaccines that were approved for use in India. Both of the vaccines were proven for their safety. The former has shown an effectiveness of $67 \%$ in reducing infections and $70 \%$ in reducing transmission $^{15}$, and we assume the same for the latter. We further assume an $80 \%$ reduction in age-dependent mortality due to vaccination ${ }^{16,17}$. The combined production capacity of these two vaccines is around 60 million doses monthly. A step up in production is expected between July and September 2021, but we present our model based on the foreseeable situation in mid-April.

COVID-19 incidence: Flu-like infections spread rapidly in winter. In the winter of 2020, India was in an 'unlock' phase with minimal NPIs, and yet COVID-19 infections were contained. Several hypotheses surrounded this observation, including protection from previous infectious diseases and herd immunity with a large unmonitored COVID-19 infection. However, in the spring of 2021, the second wave of COVID-19 started, with caseloads exceeding 150,000 daily infections (https://www.covid19india.org). The rising pattern appears to parallel the rise of infections in the spring and summer of 2020. If this situation continues, either because there is an inherent spread of infection under hot conditions due to climatization ${ }^{18}$ or due to any other reasons, one may expect rising infections until September 2021.
Typically one uses susceptible-infected-recovered $(\mathrm{SIR})^{19}$ or susceptible-asymptomatic-infected-recovered $(\mathrm{SAIR})^{20}$ models to make predictions of COVID-19 incidence. In fact, much simpler extrapolations based on global trends were also successful in making reliable predictions for a few weeks ${ }^{21}$. However, in order to forecast for many months, one has to assume various conditions that allow COVID-19 to spread - for example, the transmission rate or $R_{0}$, how it will be managed by lockdowns. Since making an assumption is unavoidable, our assumption for the second wave was guided by the global trends in the peak of infections and duration of the second wave. The motivation for this assumption was that unless the susceptible population is completely exhausted, the peak is ultimately defined by the lockdowns as a response to a breakdown of the healthcare infrastructure.

Studying the second wave from the countries ${ }^{22}$ which had the most infections (Supplementary Table 1), it is clear that all of them had a peak of the second wave that was worse than the first wave in the spring of 2020 (ratio: $4.35 \pm 1.95$ ). Several factors may have contributed to this large second peak in most countries, including the need to keep their economies active as well as their confidence in better management of critical cases through effective treatment. Further, the fullwidth at halfmaximum (a modelfree way of defining the width) for the duration of the second wave in most COVID-19-affected countries lasted 27 to 122 days, with extreme and moderate restrictive NPIs respectively. This duration is also commensurate with the width of halfmaximum (108 days) of the first wave of COVID-19 infections which occurred in the same season in 2020. While we acknowledge that it is difficult to predict whether the actual trends in infections which may be checked by several unknowns such as attainment of herd immunity, or imposing different grades of lockdown, the global trends in the peak and width of the second wave serve as a guideline for estimating the consequences under some realistic worst-case scenarios (see below).

Age demographics: The age-binned population data were obtained from the 2011 census. To estimate the distribution in 2021 , the population in each bin was multiplied by 1.125 to match the overall $12.5 \%$ increase in the Indian population between 2011 and 2021. It is evident that the population is largely young: 553 million (0-20 yrs), 527 million (20-45 yrs), 173 million (45-60 yrs), 119 million $(60+y r s)$, with a low individual risk and requires multiple layers of prioritization.

\section{Population-level model}

Focus: The focus of this study is on how vaccination of the adults reduces the secondary infections and deaths among the seniors. Deaths among the adults and seniors 
(a)

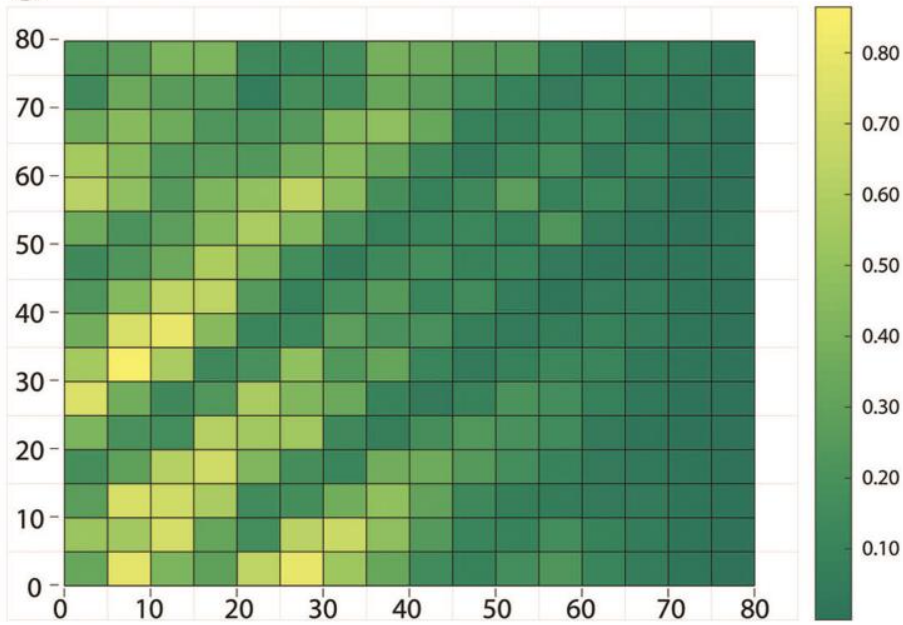

(b)

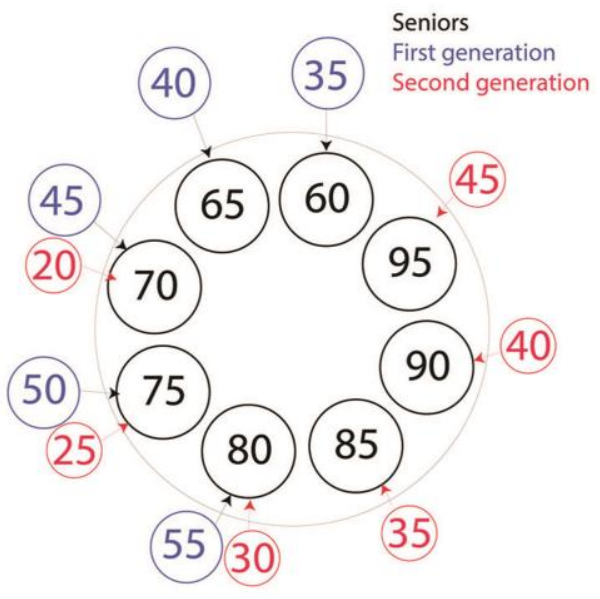

Figure 1. Intergenerational contact structure. $\boldsymbol{a}$, Age-structured home contact network in India, obtained from Prem et al. ${ }^{23}$. The figure shows a clear banded structure, with a $25-\mathrm{yr}$ intergenerational gap. The colour bar indicates the estimated mean number of contacts made by an individual ${ }^{23}$. $\boldsymbol{b}$, The inferred intra-home contact network that was used in this work. The model assigns to every senior of age $S>60 \mathrm{yrs}$, a first-generation home contact of S-25 and a second-generation home contact of S-50, as long as these contacts have an age of less than 60 yrs.

who were infected by means other than through families are not analysed or reported.

Contact structure: The home-contact matrix for India ${ }^{23}$ shows a banded structure with contact between the same age group (Figure $1 \mathrm{a}$ ), and those with difference of 25 and 50 years, suggesting an underlying generational gap of 25 years. We assume each senior of age $S$ is related to one child of age (S-25) and one grandchild of age (S-50) (Figure $1 b$ ). Since the focus is on intergenerational infections, this assignment is performed only if the age of the child or the grandchild is $<60$ years.

Overall incidence: We consider four different scenarios of the development of COVID-19 second wave in India.

The number of daily infections crossed 150,000 by 12 April 2021. We considered four different scenarios starting from this. Scenario 1 was meant to be conservative, with infections reaching a peak of 225,000 with flattening for a month and then a subsequent decrease. In scenarios $2-4$, we considered a peak daily infection of 400,000 , commensurate with the average ratio of the peak in the second versus the first waves in the top COVID-19affected countries. Similarly, guided by the global average of the full width at half peak with and without lockdowns (Supplementary Table 1), we assumed widths of around 60, 90 and 120 days for scenarios 2, 3 and 4 respectively, of the second wave in India. Based on these assumptions, Figure $2 a$ shows the four different epidemiological scenarios graphically. While assumptions of the epidemiological scenarios in a model-independent way may seem ad hoc, qualitatively these are not very different from the ad hoc assumptions of the sequence of increase or decrease in $R_{0}$ in the SIR/SAIR models.

The total number of daily infections is assumed to be distributed in the population in the ratio of the susceptible population in each bin. The overall incidence so assumed serves to define the baseline infection rate for the unvaccinated adults. However, the infections in the model differ from this. Among the adults, the baseline number is corrected by the vaccinated fraction and the chances of infection despite vaccination. Similarly, the infections transmitted by the adults to the seniors also change due to either or both of them being vaccinated.

Secondary attack rate: The average secondary attack due to a primary infection in the family is around $30 \%$ (ref. 24).

Mortality: Since we examine the age-based vaccine rollout strategy, COVID-19 mortality is attributed to the individual risk posed by the age $\mathrm{e}^{11,12}$. People with comorbidities that increase the risk of COVID-19 fatality are considered to be vaccinated early on and independent of the age-based roll-out strategy. In the absence of the relevant data, it is assumed that the age-dependent mortality does not change with the variants.

Effect of vaccination: The effect of vaccination is assumed mainly based on the data from AstraZeneca reports - the chance of infection is reduced by $67 \%$, transmission by $70 \%$ and mortality among the infected by $80 \%$. We assume that these reductions are universally applicable to all age groups and regardless of the vaccine type. 

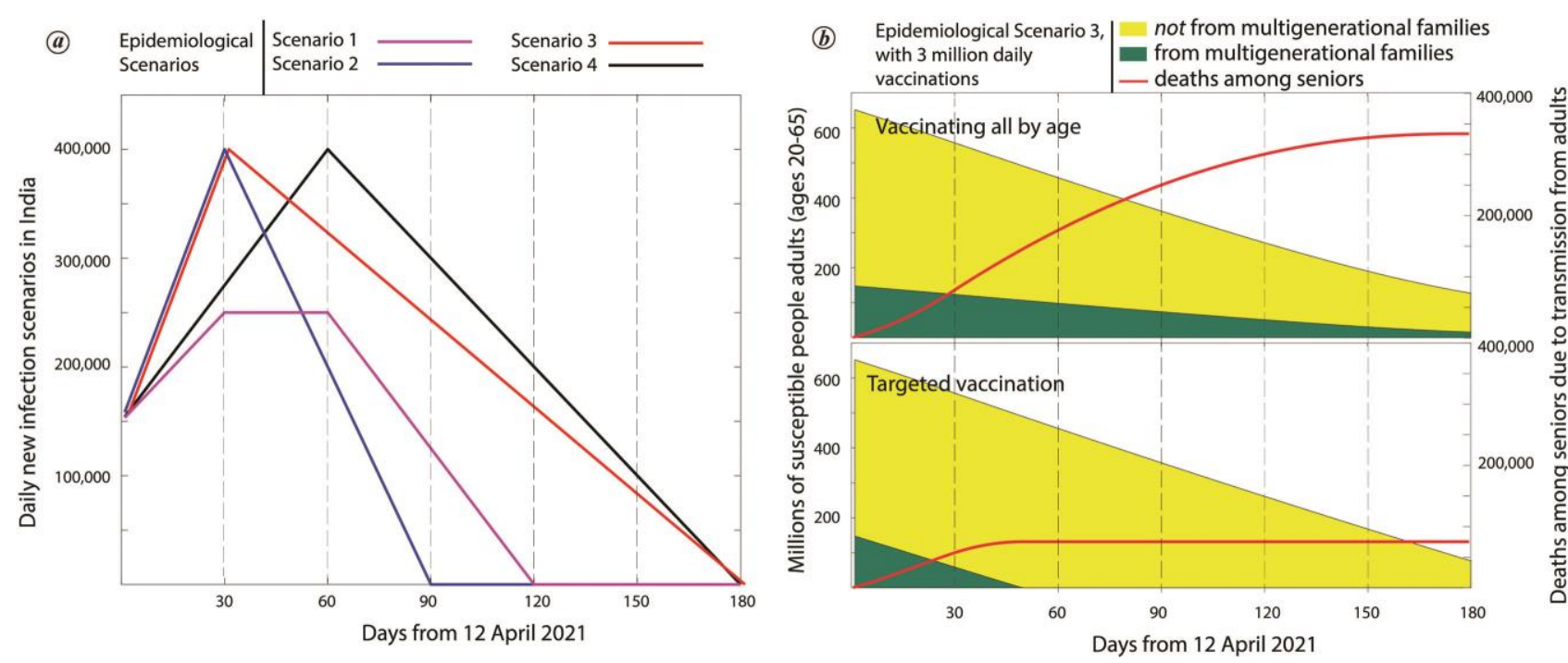

Figure 2. COVID-19 development scenarios and deaths avoided. $\boldsymbol{a}$, The four COVID-19 development scenarios considered in this study. Scenario 1 is the most conservative one considering the daily infections on 12 April 2021. The peak of daily infections in all other scenarios is assumed to be 400,000 and is based on the average ratio of second to first peak observed in countries with the most inf ections (Supplementary Table 1). $\boldsymbol{b}$, Comparison of two strategies based on (top) no selectivity in vaccinating members of multigenerational families and (bottom) preferential vaccination to younger members of multigenerational families for scenario 3 . Table 1 shows the reduction in deaths among the seniors by vaccinating the adults in all four scenarios.

Other model assumptions: The many existing and possible new variants are considered not to be different with respect to the fatality rates or for the effectiveness of the vaccine. At least in the six-month period, a person who is infected with COVID-19 is assumed not to be reinfected. The potential deaths in seniors are assigned to the day on which an adult is infected for simplicity of the analysis, although the same analysis may equally be presented with a time delay, including that of hospitalization. The vaccination rates may increase slightly: this may be compensated by the need for the second dose. Thus, to not invoke complicated scenarios of vaccine availability, hesitancy and second dose delays, we performed the analysis considering that the first dose of the vaccine implicitly delivers its effectiveness.

Calculations: Briefly, the calculations involve three different aspects (Supplementary Scheme 1).

Initialization - the demographics of the population and assignment of some of the adults to multigenerational homes were performed while maintaining the intergenerational gap of 25 years. The vaccination status of the adults and seniors is defined based on the reported coverage (https://dashboard.cowin.gov.in/).

Infection and vaccination - the evolution of the COVID-19 case load, feasible vaccination rate and choice of a targeted strategy and vaccination status of the adults was updated on a daily basis in the model. Those vaccinated as well as infected were removed from the susceptible population.

Consequences to seniors - only the infections transmitted from adults to seniors in multigenerational families were considered. The transmission, contraction and death rates are all influenced by the vaccination.

The calculations were performed on this population over a period of 180 days. The complete analysis code is available online (https://github.com/meherpr/vaccinating-young).

\section{Impact of prioritizing adults in a multigenerational home}

By 12 April 2021 (Supplementary Table 2), the priority vaccination for seniors had a coverage of around 50\%, and about $20 \%$ from the second priority age group of 45-60 years had been vaccinated (https://dashboard.cowin. gov.in/). Under the four scenarios of COVID-19 second wave development (Figure $2 a$ ), we studied the effectiveness of vaccinating with two different strategies-agebased roll-down to younger populations, and a preferential targeted vaccination of younger members from multiagenerational homes. By adopting a targeted strategy, the susceptible adults from multigenerational families may all be vaccinated in a relatively short time of 52 days if administered at 2 million doses per day, compared to a uniform age-based strategy (Figure $2 b$ ). In a more realistic scenario, the number of deaths among the seniors due to transmission from adults in the family is likely to be reduced by over 100,000 due to this targeted strategy (Table 1).

\section{Interpreting through effective risk and effective age}

While the quantitative effectiveness of vaccinating adults from multigenerational homes differs in the various 
Table 1. Reduction of deaths among seniors. The two different vaccination strategies employed have varying impacts on the transmission of infections from adults to seniors, and the consequent deaths among seniors. Results from the model are shown u nder the different pandemic scenarios. Also, we assume the age-dependent mortality rate obtained from the meta-analysis of Levin et al. ${ }^{11}$. The lives saved are also considerably high even if the mortality rates may be $20 \%$ less in the second wave in India, due to better therapeutic management or other reasons

\begin{tabular}{|c|c|c|c|c|}
\hline \multirow[b]{2}{*}{$\begin{array}{l}\text { Pattern of daily new infections } \\
\text { for } 180 \text { days }\end{array}$} & \multicolumn{2}{|c|}{$\begin{array}{l}\text { Deaths among seniors due to transmission } \\
\text { from adults. Vaccinating } 2 \text { million/day }\end{array}$} & \multicolumn{2}{|c|}{$\begin{array}{l}\text { Deaths among seniors due to transmission } \\
\text { from adults. Vaccinating } 3 \text { million/day }\end{array}$} \\
\hline & $\begin{array}{c}\text { Uniform } \\
\text { vaccination }\end{array}$ & $\begin{array}{l}\text { Targeted vaccination of } \\
\text { adults }(20-60 \mathrm{yrs})\end{array}$ & $\begin{array}{c}\text { Uniform } \\
\text { vaccination }\end{array}$ & $\begin{array}{l}\text { Targeted vaccination of } \\
\text { adults }(20-60 \mathrm{yrs})\end{array}$ \\
\hline Scenario 1 & 189,652 & 85,513 & 187,901 & 54,871 \\
\hline Scenario 2 & 183,443 & 109,663 & 182,510 & 75,077 \\
\hline Scenario 3 & 343,988 & 119,905 & 333,952 & 76,732 \\
\hline Scenario 4 & 363,405 & 102,709 & 350,929 & 60,460 \\
\hline
\end{tabular}

Table 2. Effective age. The 'effective risk' for each adult in the age group 20-55 yrs living in a multigenerational home, is defined based on the COVISHIELD (AstraZeneca) effectiveness and a 25-yr intergenerational gap. The age-dependent infection fatality rate shown in the Supplementary $\underline{\text { Table } 3}$ was obtained from Levin et al. ${ }^{11}$. The 'effective age' was obtained by a reverse-lookup of the risk from the Supplementary Table 3 . Considering the goal of reducing COVID-19 related deaths, an adult living with an unvaccinated parent or grandparent is effectively older by 15 or 40 years respectively

\begin{tabular}{|c|c|c|c|c|c|c|c|c|}
\hline \multirow[b]{2}{*}{$\begin{array}{l}\text { Age of an } \\
\text { unvaccinated adult }\end{array}$} & \multicolumn{2}{|c|}{$\begin{array}{l}\text { If with a vaccinated } \\
\text { grandparent }\end{array}$} & \multicolumn{2}{|c|}{$\begin{array}{l}\text { If with an unvaccinated } \\
\text { grandparent }\end{array}$} & \multicolumn{2}{|c|}{$\begin{array}{c}\text { If with a vaccinated } \\
\text { parent }\end{array}$} & \multicolumn{2}{|c|}{$\begin{array}{c}\text { If with an unvaccinated } \\
\text { parent }\end{array}$} \\
\hline & $\begin{array}{l}\text { Effective } \\
\text { risk }\end{array}$ & $\begin{array}{l}\text { Effective } \\
\text { age }\end{array}$ & $\begin{array}{l}\text { Effective } \\
\text { risk }\end{array}$ & $\begin{array}{l}\text { Effective } \\
\text { age }\end{array}$ & $\begin{array}{l}\text { Effective } \\
\text { risk }\end{array}$ & $\begin{array}{l}\text { Effective } \\
\text { age }\end{array}$ & $\begin{array}{l}\text { Effective } \\
\text { risk }\end{array}$ & $\begin{array}{l}\text { Effective } \\
\text { age }\end{array}$ \\
\hline 20 & 0.05392 & 35 & 0.7984 & 60 & 0.00874 & 20 & 0.0454 & 35 \\
\hline 25 & 0.09628 & 40 & 1.4245 & 65 & 0.01546 & 25 & 0.0775 & 40 \\
\hline 30 & 0.16634 & 45 & 2.459 & 70 & 0.02756 & 30 & 0.146 & 45 \\
\hline 35 & 0.31864 & 50 & 4.684 & 75 & 0.05386 & 35 & 0.271 & 52 \\
\hline 40 & 0.56716 & 55 & 8.356 & 80 & 0.09574 & 42 & 0.499 & 56 \\
\hline 45 & 0.62716 & 55 & 8.416 & 80 & 0.17752 & 48 & 0.922 & 62 \\
\hline 50 & 0.71716 & 60 & 8.506 & 80 & 0.30478 & 53 & 1.633 & 67 \\
\hline 55 & - & - & 8.506 & - & 0.56634 & 57 & 2.859 & 71 \\
\hline
\end{tabular}

epidemiological scenarios, the advantage remains in all of them and may be interpreted in a simple way by defining the 'effective risk'. Age and the presence of comorbidities are individual risk factors for COVID-19 fatality ${ }^{11,12}$. The risk profile of an individual is defined by the infection fatality rate (IFR), which quantifies the chance of death when a person of a given demographic is infected by COVID-19. Defining an adult living in a multigenerational home as a new demographic allows us to obtain an effective risk profile for this group. The chance of death in this adult-senior duo, due to a COVID-19 infection in the adult, is the chance of fatality in the adult combined with the chance of fatality in the senior weighted by the chance of secondary attack (transmission) at home.

Effective risk (adult) $=$ IFR (adult) + secondary attack rate $*$ chance of infection of a vaccinated or unvaccinated senior $*$ mortality risk of a vaccinated or an unvaccinated senior.

This definition is conditional to the assumption that there is a person with a primary infection in the household, similar to the definition of secondary attack rate conditional to a primary infection. We used the infection fatality rate data (Supplementary Table 3 ) from Levin et $a l .{ }^{11}$ to calculate the effective risk and estimate the effective age (Table 2). Table 2 utilizes the home contact structure for India with an approximate 25-yr intergenerational gap, and the effectiveness of the COVISHIELD vaccines that are mostly used in India. It is evident that the effective age of an adult in a multigenerational home is 15 years more if living with an unvaccinated parent (senior) or 40 years more if living with an unvaccinated grandparent (senior). This personal risk quantification clarifies our estimates of the advantages of preferential vaccination of adults from multigenerational homes as a public health strategy.

\section{Possible immediate strategy for prioritization in India}

Vaccinating family members of those vulnerable is a well-known strategy to break the transmission chain leading up to the vulnerable. This is especially recommended if the vaccine is not safe for the vulnerable, or if they are 
not likely to develop antibodies due to a compromised immune system. Multigenerational homes are considered to be the major factor behind the high COVID-19 casualties in many countries; Italy, for example. Whereas in the vaccination strategies of $\mathrm{USA}^{10}$, the considerations of multigenerational homes has been important mainly to identify those vulnerable outside the long-term care facilities. The Swiss taskforce explicitly prioritized 'close contacts', household members or relatives providing care to people at risk, in priority group 3. However, the rationale, the age groups which qualify and comparison between individuals of middle age versus those who are younger but living with older members could not be gathered easily from the public domain. Further, to the best of our knowledge, public health strategies weighing the epidemiological scenarios against vaccine availability ${ }^{25}$ have not evaluated the societal impact of vaccinating family members of those vulnerable, considering the local realities of the family structure, secondary attack rates and effectiveness of the available vaccines. We have devised a strategy based on the effective risk and quantified its effectiveness using our model-based analysis. This effective risk or effective age among people of low individual risk may be extended to different demographics by combining their familial, social or work-related interactions.

The vaccination drive in India is active, and ranks very high globally in absolute numbers (132 million doses administered as of 12 April 2021). Although 41 million seniors and 91 million adults have been vaccinated, there are still large gaps in coverage (Supplementary Table 2). Delhi has a vaccination coverage of $53 \%$ among the seniors. The coverage among seniors varied widely across the states with a mean and standard deviation of $42 \%$ and $21 \%$ respectively. We ask if vaccinating the vulnerable, and those next to them, is easily achievable with the available resources. The number of vaccines required to completely vaccinate the seniors is around 77 million more. Similarly, from our estimates based on the family structure model we have described, the number of adults from multigenerational homes that need to be vaccinated is 144 million more. Paradoxically, the two major factors that pose a significant hurdle to effective vaccination programmes are the shortage of vaccines and hesitancy among some of those eligible. Interestingly, one of the largest States Rajasthan has achieved a vaccination coverage of $78 \%$ among the Seniors, setting a precedence for allowing other States to follow a similar model. Thus, with the stock of vaccinations India has, it is feasible to vaccinate almost all of these high risk and high effective risk populations within 2-3 months.

\section{Limitations and future work}

The calculations were based on the epidemiological and vaccination scenarios that seemed plausible. However, this situation may change dynamically with access to newer sources of vaccines. The work assumed a 25-yr intergenerational gap, that we inferred from the banded contact network of Prem et al. ${ }^{23}$ Other scenarios such as fully senior households were not considered, assuming that the model nevertheless captures a majority of the family structure. However, we also consider that by including contact with domestic help in some cases, the risk exposure to seniors through adults may be equivalent to having younger family members at home. A study based on more granular family structured data, if available, will be interesting to recalibrate the effectiveness of the strategy we propose here.

Needless to say, there is a scope for examining several other strategies in greater detail, such as by recognizing that the pandemic spread is not uniform across the country, and the case load migrates ${ }^{26}$, it is also possible to preferentially vaccinate the populations with high risk and high effective risk in districts or States that are at the highest risk of COVID-19 infections. Similarly, the twopronged strategy of keeping the infections under control with lockdowns in the selective hotspots and vaccinating those regions rapidly.

\section{Conclusion}

While successfully conducting large-scale vaccination drives, India faces the challenge of vaccinating a large young population. The effective age we defined for an unvaccinated adult living with a grandparent by combining their risks, may be even 40 years more, thus underscoring the need to vaccinate those living with the vulnerable. By placing this risk through the adults in the family, in the context of current vaccination rates, it is possible to prioritize and administer the vaccines to the seniors and adults who are in multigenerational homes, thereby significantly reducing the chances of deaths in epidemiological scenarios where the second wave may last for an additional 3-6 months.

Scripts. The scripts used in this analysis are available at https://github.com/meherpr/vaccinating-young

1. Ferguson, N. et al., Report 9: Impact of non-pharmaceutical interventions (NPIs) to reduce COVID19 mortality and healthcare demand. Imperial College London, 2020, pp. 491-497, 10.77482.

2. Randolph, H. E. and Barreiro, L. B., Herd immunity: understanding COVID-19. Immunity, 2020, 52(5), 737-741.

3. Fontanet, A. and Cauchemez, S., COVID-19 herd immunity: where are we? Nature Rev. Immunol., 2020, 20(10), 583-584.

4. Anderson, R. M. et al., How will country-based mitigation measures influence the course of the COVID-19 epidemic? The Lancet, 2020, 395(10228), 931-934.

5. Corum, J. et al., Coronavirus vaccine tracker. The New York Times, 2020, p. 5.

6. Forni, G. and Mantovani, A., COVID-19 vaccines: where we stand and challenges ahead. Cell Death Different., 2021, 28(2), 626639. 
7. Voysey, M. et al., Single-dose administration and the influence of the timing of the booster dose on immunogenicity and efficacy of ChAdOx1 nCoV-19 (AZD1222) vaccine: a pooled analysis of four randomised trials. The Lancet, 2020, 397(10277), 881-891.

8. Mills, M. C. and Salisbury, D., The challenges of distributing COVID-19 vaccinations. EClinicalMedicine, 2021, 31

9. Irwin, A., What it will take to vaccinate the world against COVID19. Nature, 2021, 592(7853), 176-178.

10. National Academies of Sciences, Engineering, and Medicine. Framework for equitable allocation of COVID-19 vaccine, 2020

11. Levin, A. T. et al., Assessing the age specificity of infection fatality rates for COVID-19: systematic review, meta-analysis, and public policy implications. Eur. J. Epidemiol., 2020, 35, $1123-1138$.

12. Ghisolfi, S. et al., Predicted COVID-19 fatality rates based on age, sex, comorbidities and health system capacity. BMJ Global Health, 2020, 5(9), e003094.

13. Public Health England, JCVI recommends that adults living with adults who are immunosuppressed should be prioritised for the COVID-19 vaccine, 29 March 2021; https://www.gov.uk/ government/news/new-vaccine-advice-for-adults-living-with-adultswho-are-immunosuppressed

14. Ella, R. et al., Safety and immunogenicity of an inactivated SARS-CoV-2 vaccine, BBV152: interim results from a doubleblind, randomised, multicentre, phase 2 trial, and 3 -month followup of a double-blind, randomised phase 1 trial. The Lancet Inf. Dis., 2021, 21, 637-646.

15. Voysey, M. et al., Safety and efficacy of the ChAdOx1 nCoV-19 vaccine (AZD1222) against SARS-CoV-2: an interim analysis of four randomised controlled trials in Brazil, South Africa, and the UK. The Lancet, 2021, 397(10269), 99-111.

16. Bernal, J. L. et al., Early effectiveness of COVID-19 vaccination with BNT162b2 mRNA vaccine and ChAdOx1 adenovirus vector vaccine on symptomatic disease, hospitalisations and mortality in older adults in England. medRxiv, 2021; https://doi.org/10.1101/ 2021.03.01.21252652.

17. Public Health England, New data show vaccines reduce severe COVID-19 in older adults, 1 March 2021; https://www.gov.uk/ government/news/new-data-show-vaccines-reduce-severe-covid19-in-older-adults

18. Sruthi, C. K. et al., Estimating Effect-sizes to Infer if COVID-19 transmission rates were low because of masks, heat or high because of air-conditioners, Tests. medRxiv, 2021.

19. Kermack, W. O. and McKendrick, A. G., Contributions to the mathematical theory of epidemics-I. Proc. R. Soc. London, Ser. A, 1932, 138(834) 55-83.

20. Kaushal, S. et al., Estimating the herd immunity threshold by accounting for the hidden asymptomatics using a COVID-19 specific model. PLoS ONE, 2020, 15(12), e0242132.

21. Prakash, M. K. et al., Minimal and adaptive numerical strategy for critical resource planning in a pandemic. Phys. Rev. E, 2020, 102(2), 021301.

22. Worldometer. COVID-19 pandemic, 2020; https://www.worldometers.info/coronavirus/\#countries

23. Prem, K., Cook, A. R. and Jit, M., Projecting social contact matrices in 152 countries using contact surveys and demographic data. PLoS Comput. Biol., 2017, 13(9), e1005697.

24. Madewell, Z. J. et al., Household transmission of SARS-CoV-2: a systematic review and meta-analysis. JAMA Network Open, 2020, 3(12), e2031756-e2031756.

25. Bubar, K. M. et al., Model-informed COVID-19 vaccine prioritization strategies by age and serostatus. Science, 2021, 371(6532), 916-921.

26. Ansumali, S. et al., A steady trickle-down from metro districts and improving epidemic-parameters characterize the increasing COVID-19 cases in India. 2020; available at SSRN 3701553.

ACKNOWLEDGEMENT. S.A. and M.K.P. thank Dr Aloke Kumar for helpful discussions.

Received and accepted 25 May 2021

doi: $10.18520 / \mathrm{cs} / \mathrm{v} 120 / \mathrm{i} 11 / 1698-1704$ 